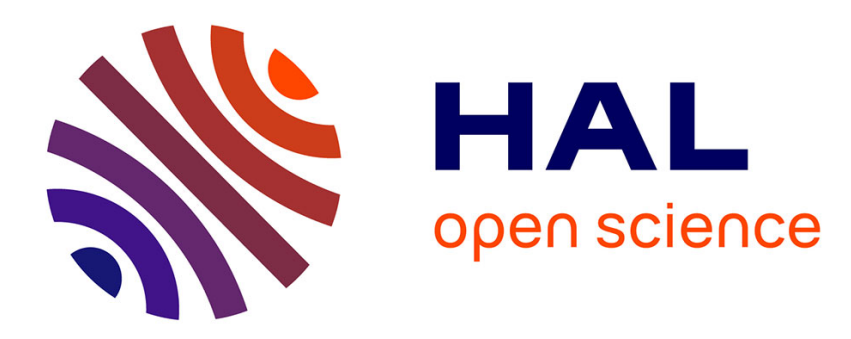

\title{
Etude de la propagation dans un fluide par la méthode des volumes finis
}

\author{
M. Boheas, V. Martin
}

\section{To cite this version:}

M. Boheas, V. Martin. Etude de la propagation dans un fluide par la méthode des volumes finis. Journal de Physique IV Proceedings, 1994, 04 (C5), pp.C5-725-C5-728. 10.1051/jp4:19945156 jpa00252835

\section{HAL Id: jpa-00252835 https://hal.science/jpa-00252835}

Submitted on 1 Jan 1994

HAL is a multi-disciplinary open access archive for the deposit and dissemination of scientific research documents, whether they are published or not. The documents may come from teaching and research institutions in France or abroad, or from public or private research centers.
L'archive ouverte pluridisciplinaire HAL, est destinée au dépôt et à la diffusion de documents scientifiques de niveau recherche, publiés ou non, émanant des établissements d'enseignement et de recherche français ou étrangers, des laboratoires publics ou privés. 


\title{
Etude de la propagation dans un fluide par la méthode des volumes finis
}

\author{
M.A. BOHEAS et V. MARTIN*
}

Laboratoire de Mécanique et d'Acoustique, CNRS, 13402 Marseille cedex 20, France

* IMT, 13451 Marseille cedex, France

\begin{abstract}
The study of temporal acoustic problems, linear or not, can be carried out by solving NavierStokes equations. The finite volume method is chosen in which the compressibility is introduced by the law of behaviour. In that case, constant isentropy is taken into account. After a short description of the basic equations, a 1D example shows how is used the boundary condition of radiation at infinity for the plane wave. Added to the calculation process a feedback loop has to control a secondary acoustic source for active noise cancellation.
\end{abstract}

\section{Préambule}

La modélisation numérique en acoustique linéaire harmonique a permis d'avoir des renseignements "statiques" pour la position optimale des contre-sources lors contrôle acoustique actif [1]. Ces positions n'ont de sens qu'en régime établi. Que se passera-t-il lors de changements de régimes? La modélisation temporelle est un passage obligé. Le présent travail décrit la démarche choisie ainsi qu'une application élémentaire au contrôle actif. On peut noter que cette approche n'est pas tellement répandue alors que le besoin s'en fait sentir.

\section{Méthodologie}

Le problème à traiter est un problème de couplage écoulement acoustique. L'écoulement est régi par les équations de Navier-Stokes:

- équation de continuité :

$$
\frac{\partial \rho}{\partial t}+\frac{\partial\left(\rho u_{i}\right)}{\partial x_{i}}=\mathbf{o}
$$

- équation de quantité de mouvement :

$$
\frac{\partial\left(\rho u_{i}\right)}{\partial t}+\frac{\partial\left(\rho u_{i} u_{j}\right)}{\partial x_{j}}=-\frac{\partial p}{\partial x_{i}}-\frac{\partial}{\partial x_{i}}\left(\frac{2 \mu}{3} \frac{\partial u_{k}}{\partial x_{k}}\right)+\frac{\partial}{\partial x_{j}} \mu\left(\frac{\partial u_{i}}{\partial x_{j}}+\frac{\partial u_{j}}{\partial x_{i}}\right)
$$

Les équations de continuité et de quantité de mouvement peuvent se mettre sous la forme plus générale de l'équation dite de convection-diffusion (forme générale motivée par l'utilisation ultérieure de l'algorithme de résolution la traitant [2][3][4]) : 


$$
\underbrace{\frac{\partial(\rho \Phi)}{\partial t}}_{\text {instationnarité }}+\underbrace{\frac{\partial\left(\rho u_{j} \Phi\right)}{\partial x_{j}}}_{\text {convection }}=\underbrace{\frac{\partial}{\partial x_{j}}\left(\Gamma \frac{\partial \Phi}{\partial x_{j}}\right)}_{\text {diffusion }}+\underbrace{S_{i}}_{\text {srurce }}
$$

La continuité correspond à $\boldsymbol{\Phi}=\mathbf{1}, \boldsymbol{\Gamma}=\mathbf{0}$, et $\boldsymbol{S}=\mathbf{0}$. L'identification des termes de l'équation de quantité de mouvement conduit à :

$$
\underbrace{\frac{\partial\left(\rho u_{i}\right)}{\partial t}}_{\text {instationnarité }}+\underbrace{\frac{\partial\left(\rho \boldsymbol{u}_{i} \boldsymbol{u}_{j}\right)}{\partial \boldsymbol{x}_{j}}}_{\text {convection }}=\underbrace{\frac{\partial}{\partial x_{j}}\left(\mu \frac{\partial u_{i}}{\partial \boldsymbol{x}_{j}}\right)}_{\text {diffusion }}+\underbrace{\frac{\partial}{\partial \boldsymbol{x}_{j}}\left(\mu \frac{\partial u_{j}}{\partial x_{i}}\right)-\frac{\partial}{\partial x_{i}}\left(\frac{2 \mu}{3} \frac{\partial u_{k}}{\partial x_{k}}\right)-\frac{\partial p}{\partial x_{i}}}_{\text {terme source }}
$$

avec $\phi=\boldsymbol{u}_{i}$. Les équations de Navier-Stokes peuvent donc se mettre sous la forme proposée. Notons que le terme source de la quantité de mouvement inclut le gradient de pression.

La résolution de l'équation générale de la diffusion

$$
\frac{\partial(\rho \Phi)}{\partial t}+\frac{\partial}{\partial x_{j}}\left(\rho u_{j} \Phi-\Gamma \frac{\partial \Phi}{\partial x_{j}}\right)=S
$$

par la méthode des volumes finis s'exprime sur un volume de contrôle $2 \mathrm{D}$ (figure 1), sous la forme discrétisée suivante :

$$
a_{P} \Phi_{P}=a_{E} \Phi_{E}+a_{W} \Phi_{W}+a_{N} \Phi_{N}+a_{S} \Phi_{S}+b
$$

Ce qui conduit à l'établissement d'un système d'équations, la résolution du système peut être effectuée par une méthode d'élimination de GAUSS, après introduction des conditions aux limites, et éventuellement des sources correspondant au problème traité.

Notons que l'équation des ondes de Lighthill - et partant, l'équation de propagation acoustique est obtenue en combinant $\partial(\mathbf{1}) / \partial \boldsymbol{t}$ et $\operatorname{div}(\mathbf{2})$ :

$$
\frac{\partial^{2} \rho}{\partial^{2} t}-c_{\circ} \frac{\partial^{2} \rho}{\partial x_{i} \partial x_{i}}=\frac{\partial^{2}}{\partial x_{i} \partial x_{j}}\left(\rho u_{i} u_{j}+\left(p-c_{o}^{2} \rho\right) \delta_{i j}+\tau_{i j}^{\prime}\right)
$$

avec :

$$
\tau_{i j}^{\prime}=\frac{\partial}{\partial x_{j}}\left(\frac{\partial}{\partial x_{i}}\left(\frac{2 \mu}{3} \frac{\partial u_{k}}{\partial x_{k}}\right)-\frac{\partial}{\partial x_{j}} \mu\left(\frac{\partial u_{i}}{\partial x_{j}}+\frac{\partial u_{j}}{\partial x_{i}}\right)\right)
$$

La résolution de Navier Stokes conduit automatiquement à la vérification de (6), pourtant on se garde bien de résoudre directement l'équation de Lightill (6) au profit de (1) et (2) de manière à rester au plus près des équations de base.

\section{Traitement d'une onde plane guidée}

La capacité du progiciel à traiter des problèmes acoustiques est montré sur un problème $1 \mathrm{D}$ dans lequel on simule la progation d'une onde plane de $200 \mathrm{~Hz}$ dans un milieu infini. Il y a lieu d'introduire des conditions aux limites non réfléchissantes pour le modèle de dimension finje soit les conditions d'Orlanski [5] :

$$
\frac{\partial \phi}{\partial t}+c \times \frac{\partial \phi}{\partial x_{i}}=\mathbf{o}
$$


L'expression de la condition aux limites discrétisée devient:

$$
\phi_{B}^{n+1}=\phi_{B}^{n}-\frac{c \Delta t}{\Delta x}\left(\phi_{B}^{n}-\phi_{p}^{n}\right)
$$

La figure 2 donne une illustration des résultats avec introduction des conditions aux limites.

\section{Introduction d'un contrôle élémentaire}

La mise au point du programme de contrôle élémentaire a été effectuée sur un cas analytique simulant la situation suivante dans l'air :

- une source sinusoïdale de fréquence de $200 \mathrm{~Hz}$ et d'amplitude A, émet un signal primaire;

$$
S P=A * S I N U S(\omega * t)
$$

- Une source secondaire émet un signal sinusoïdal de même fréquence mais d'amplitude quelconque. La distance (d) entre les sources étant connue ainsi que la vitesse du son dans l'air, le retard de phase de la source secondaire est calculé, de sorte que les signaux soient en phase

$$
S S=B * S I N U S\left(\omega *\left(t-\frac{d}{c}\right)\right)
$$

- un point d'observation YP est situé à l'arrière de la source secondaire à une distance (D) de cette source. La vibration observée à cet endroit a pour expression:

$$
\boldsymbol{Y P}=A * \operatorname{SINUS}\left(\omega *\left(t-\frac{d+D}{c}\right)\right)+B * \operatorname{SINUS}\left(\omega *\left(t-\frac{D}{c}\right)\right)
$$

L'amplitude de vibration de la source secondaire B est modifiée jusqu'a ce que l'amplitude YP soit inférieure à un critère fixé par l'utilisateur. Cet algorithme est introduit dans le progiciel aux volumes finis. Le même type de situation que pour le cas analytique est simulé avec un modèle $2 \mathrm{D}$. Une source ponctuelle primaire SP sinusoïdale de fréquence $200 \mathrm{~Hz}$ est placée dans le milieu étudié, une source secondaire SS ponctuelle est placée derrière puis un point d'observation YP. La source secondaire doit être sinusoïdale de même fréquence, le retard de phase peut être calculé à partir de la mesure de la vitesse du son en observant YP. L'amplitude de la source secondaire est corrigée de manière à obtenir une vibration de faible amplitude au point d'observation YP.Le niveau en YP est une fonction convexe de $\mathrm{B}$. Il est donc aisé de chercher à se placer près du minimum dès que l'on est attentif à obtenir une convergence rapide. Les résultats correspondants sont reportés sur les figures 3 et 4 . La figure 3 met en évidence la possibilité de réaliser le contrôle pour une source primaire d'amplitude donnée alors que la figure 4 correspond à un contrôle avec changement de l'amplitude de la source primaire. 


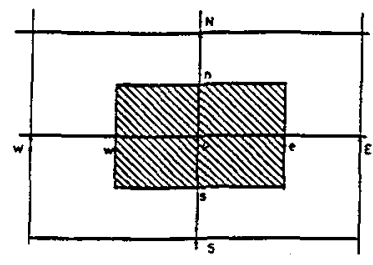

Figure - 1 - Maillage 2D

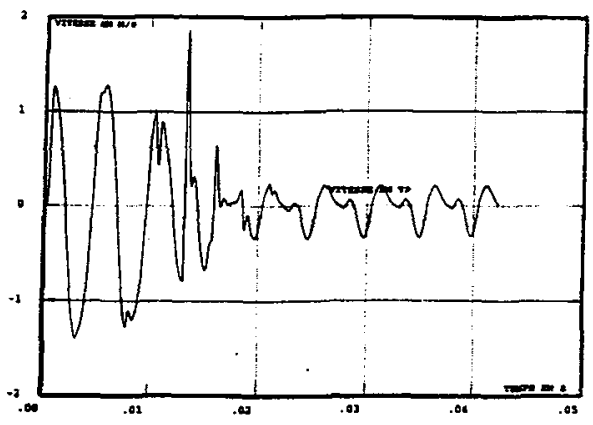

Figure - 3 - Contrôle Elementaire

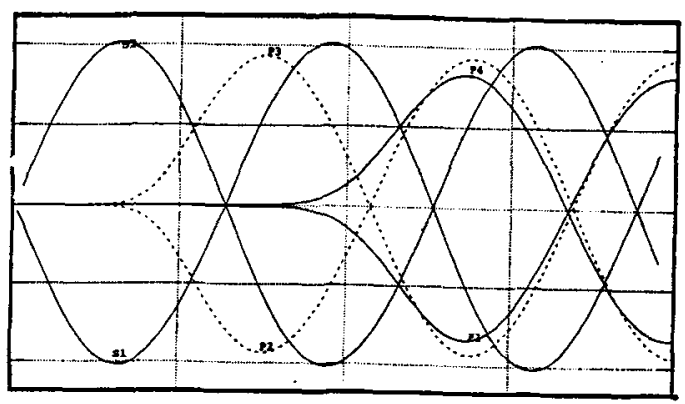

Figure - 2 - Pression $F(t)$ et $C L$

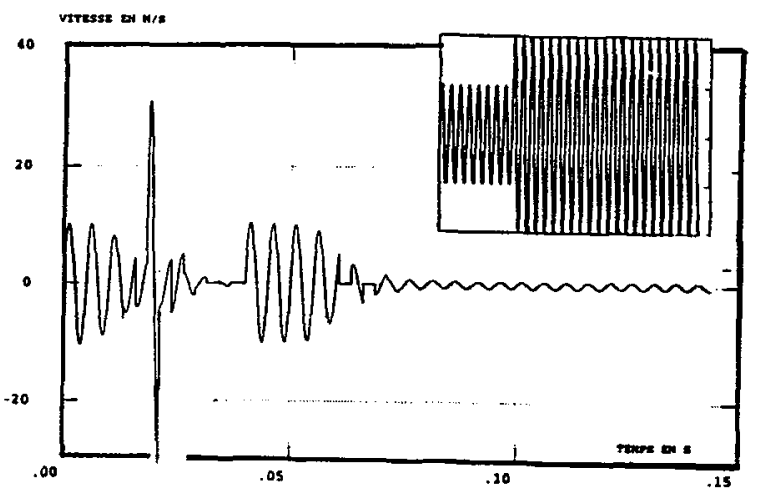

Figure - 4 - Contrôle avec changement d'amplitude

\section{Conclusion et remerciements}

On a montré la capacité d'un progiciel réalisé pour l'écoulement fluide à traiter des problèmes acoustiques dans le cadre de nos préoccupations sur le contrôle actif, en particulier via l'insertion d'un algorithme de commande dans le code. Ce travail est un maillon de la chaîne concernant la simulation numérique du contrôle.

Nous remercions la société Arcofluid IMT, distributeur du progiciel, de son intervention pour les premières compétences techniques.

\section{References}

[1] E. Benzaria,V. Martin, Détermination des Positions des Sources Secondaires en Contrôle Actif. $3^{e}$ congrès français d'acoustique Toulouse, 1994 .

[2] S. V. Patankar, Numerical Heat Transfer and Fluid Flow, Hemisphere/Mc Graw-Hill, New York, 1980 .

[3] S. V. Patankar, A Calculation Procedure for Two-Dimensionnal Elliptic Situations. J.Fluid Mechanics, vol.248, pp.267-296, 1993.

[4] D.s. Jang, R. Jetli, and S. Acharya, Comparison of the Piso, Simpler, And Simplec Algorithms for the Treatment of the Pressure-Velocity Coupling in Steady Flow Problems. Numerical Heat Transfer, vol. 10, pp. 209-228, 1986.

[5] M.H. Kobayashi, J.C.F. Pereira and J.M.M. Sousa, Comparison of Several Open Boundary Numerical Treatment for Laminar Recirculating Flows, Int. J. Numer. Methods Fluids, vol.16, pp.403-419, 1993. 